\title{
Zonation biogéographique des Alpes dauphinoises à partir de l'étude comparative des sapinières à Abies alba et des pessières à Picea abies
}

\author{
M Oberlinkels 1*, G Cadel 1, G Pautou 1, B Lachet 2 \\ 1 Université Joseph Fourier, laboratoires d'écologie végétale, BP 53X; \\ 2 CEAVCENG, département de recherche fondamentale/groupe informatique, \\ $B P$ 85X, 38041 Grenoble cedex, France
}

(Reçu le 23 février 1989; accepté le 5 avril 1990)

\begin{abstract}
Résumé - Si les biologistes s'accordent pour distinguer 3 entités phytogéographiques principales dans l'arc alpin (Alpes externes, Alpes intermédiaires, Alpes internes) cette unanimité disparaît quand il s'agit d'en fixer les limites respectives. Les auteurs abordent ce problème dans les Alpes dauphinoises, à la latitude de Grenoble. Les interprétations proposées reposent sur un traitement statistique effectué sur 274 relevés phytoécologiques et plus de 300 espèces ligneuses et herbacées. Ces relevés appartiennent à des hêtraies-sapinières et sapinières-pessières et se distribuent sur un transect W-E depuis la Chartreuse jusqu'au Briançonnais. Ce choix se justifie par le fait que le hêtre, ie sapin et l'épicéa sont largement représentés dans les Alpes dauphinoises et constituent les espèces majeures dans la plupart des peuplements montagnards.

Des AFC ${ }^{\star \star}$ et des $\mathrm{CAH}^{* * *}$ effectués sur ce corps de données ont conduit les auteurs à préciser les caractéristiques phytogéographiques de chaque type de peuplement. En outre, une analyse des relevés par la méthode de l'information mutuelle a débouché sur une caractérisation des différents secteurs et sous-secteurs à l'aide d'espèces herbacées ayant une distribution et une écologie bien affirmées.

Les auteurs discutent la validité des découpages qu'ils avaient proposés dans un travail antérieur (Cadel et Pautou, 1984) : la division des Alpes internes en 2 sous-secteurs est confirmée par les analyses multidimensionnelles; en revanche, la partition des Alpes intermédiaires en 2 soussecteurs ne s'impose pas avec la même netteté.
\end{abstract}

Abies alba / Alpes occidentales / analyse statistique / espèce indlcatrice / phytoécologie / Picea ables

Summary - Blogeographic zonation of the Dauphiné Alps based on a comparative study of Ables alba fir plantations and Picea ables spruce plantations. Biologists have distinguished 3 phytogeographical areas in the alpine range: the external Alps, intermediate and internal Alps; however, their respective limits have not yet been determined. This problem has been studied in the Dauphiné Alps, on a latitude with Grenoble.

The interpretations put forward are based on a statistical analysis of 274 phytoecological samples and over 300 herbaceous and ligneous species. These samples involved beech - fir and fir - spruce stands and were distributed along a W-E transect from Chartreuse to Briançon. This selection was

\footnotetext{
* Correspondance et tirés à part.

** AFC : analyse factorielle des correspondances.

*** $\mathrm{CAH}$ : classification ascendante hiérarchique.
} 
made due to the fact that the beech, the fir and the spruce are heavily represented in the Dauphine Alps and constitute the main species found in most mountainous populations.

FAA (factorial agreement analysis) and HAC (hierarchic ascending classification) carried out on the total data resulted in the determination of phytogeographic characteristics of each type of population. In addition, sample analysis via the mutual information method resulted in a characterization of the various sectors and subsectors using herbaceous species with a well established distribution and ecology.

The validity of the divisions which the authors had proposed in a former study is discussed (Cadel and Pautou, 1984): the division of the internal Alps into 2 subsectors is corroborated by multidimensional analysis; however, the division of the intermediate Alps into 2 subsectors is not as clear-cut.

Abies alba / western Alps / statistical analysis / indicator species / phytoecology / Picea abies

\section{INTRODUCTION}

Les phytogéographes alpins reconnaissent l'existence de 2 entités phytogéographiques majeures dans la chaîne alpine : celle des Alpes externes et celle des Alpes internes. Depuis une quarantaine d'années, plusieurs auteurs de langue germanique et notamment Kuoch (1954) ont proposé d'invidualiser une zone des Alpes intermédiaires ou Zwischenalpen dans les Alpes centrales et orientales; plus récemment Mayer (1963, 1974) a, pour les mêmes régions, réexaminé en détail cette question.

Dans les Alpes occidentales, Ozenda (1966) fut le premier à distinguer une zone des Alpes intermédiaires. Plus récemment Cadel et Pautou (1984) ont proposé pour le Dauphiné un découpage phytogéographique en 5 niveaux (fig 2), les Alpes intermédiaires et les Alpes internes étant chacune subdivisée en deux sous-secteurs. On pourrait le faire également pour les Alpes externes mais les deux soussecteurs ainsi individualisés seraient plus le reflet de caractéristiques édaphiques que climatiques; c'est la raison pour laquelle, les auteurs considèrent que les Alpes externes constituent une seule entité.
Rappelons rapidement les grandes caractéristiques phytogéographiques des 5 niveaux retenus :

\section{Alpes externes}

Elles comprennent les Préalpes calcaires et les versants ouest de Belledonne et du Taillefer sur roche mère acide. Le charme et le hêtre y trouvent leur optimum mais le sapin et l'épicéa jouent aussi un grand rôle dans la dynamique des séries.

\section{Alpes intermédiaires}

Bien représentées au niveau du bassin de Bourg d'Oisans, elles sont caractérisées par l'absence du charme dans le collinéen, la présence du hêtre, du sapin, de l'épicéa dans le montagnard; dans le subalpin, épicéa, pin à crochets et pin cembro peuvent être présents dans l'ensemble de la zone (Pautou et al, 1986). Deux sous-secteurs sont distingués.

\section{Sous-secteur ouest}

Le charme a disparu, les hêtraies sèches occupent les adrets (Gillot, 1981). Dans 


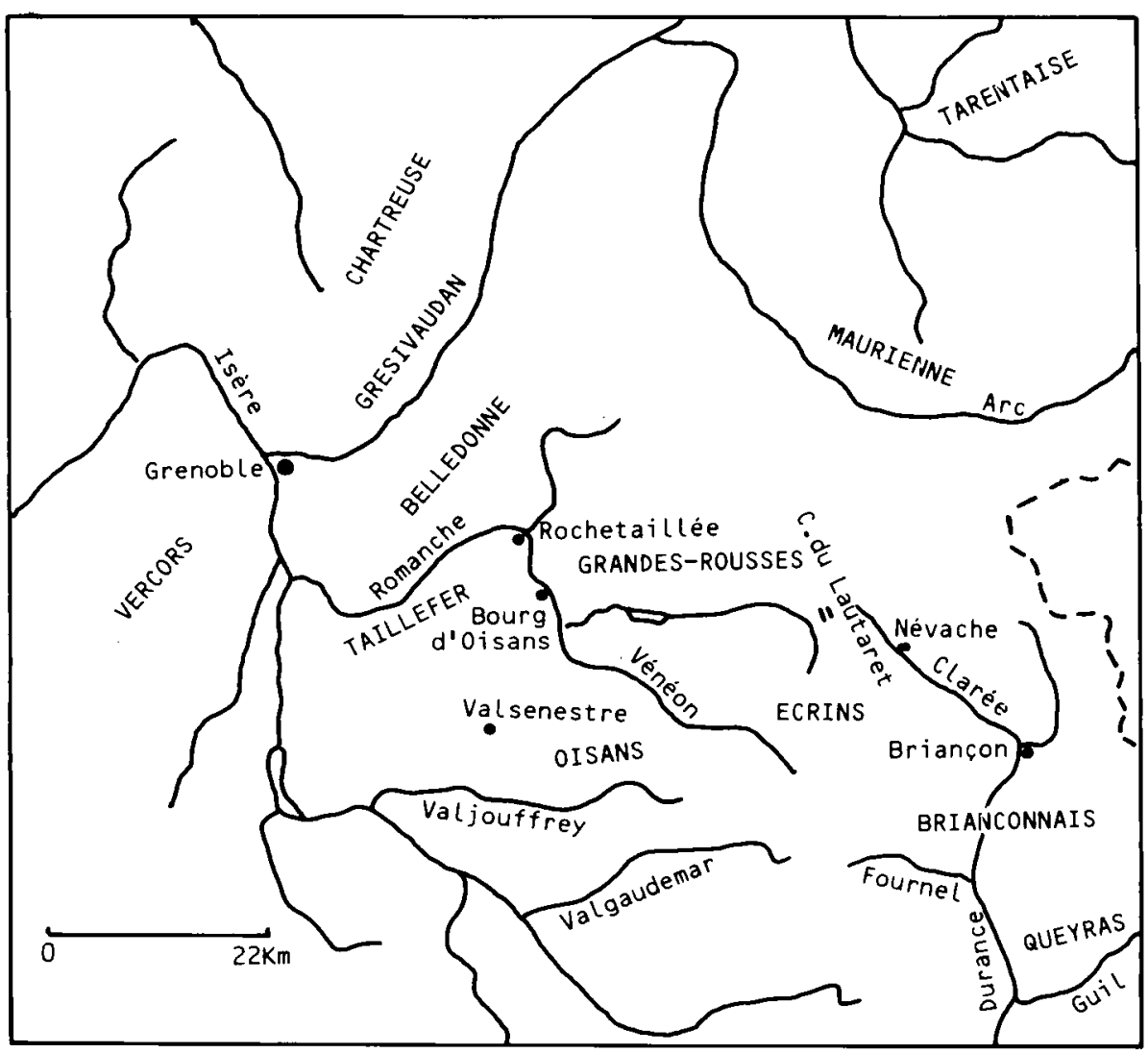

Fig 1. Carte de situation.

les ubacs le rôle principal est joué par une hêtraie-sapinière dont la composition floristique est intermédiaire entre l'AbietoFagetum et l'Abietetum albae. Les groupements subalpins sont voisins de ceux des Alpes externes sur roche mère acide, en raison notamment de l'absence du mélèze.

\section{Sous-secteur est}

L'étage collinéen étant très réduit, c'est au niveau du montagnard que ce soussecteur s'individualise le mieux. Le hêtre est rare et le pin sylvestre domine sur les adrets. En ubac, le sapin et l'épicéa forment des forêts proches de l'Abietetum albae décrit par Kuoch (1954), association encore riche en espèces des Fagetalia sy/vaticae. Le mélèze est présent dans le montagnard et le subalpin inférieur (Duchaufour et Fourchy, 1952).

\section{Alpes internes}

Elles débutent à l'est des Grandes Rousses et des premières crêtes du mas- 


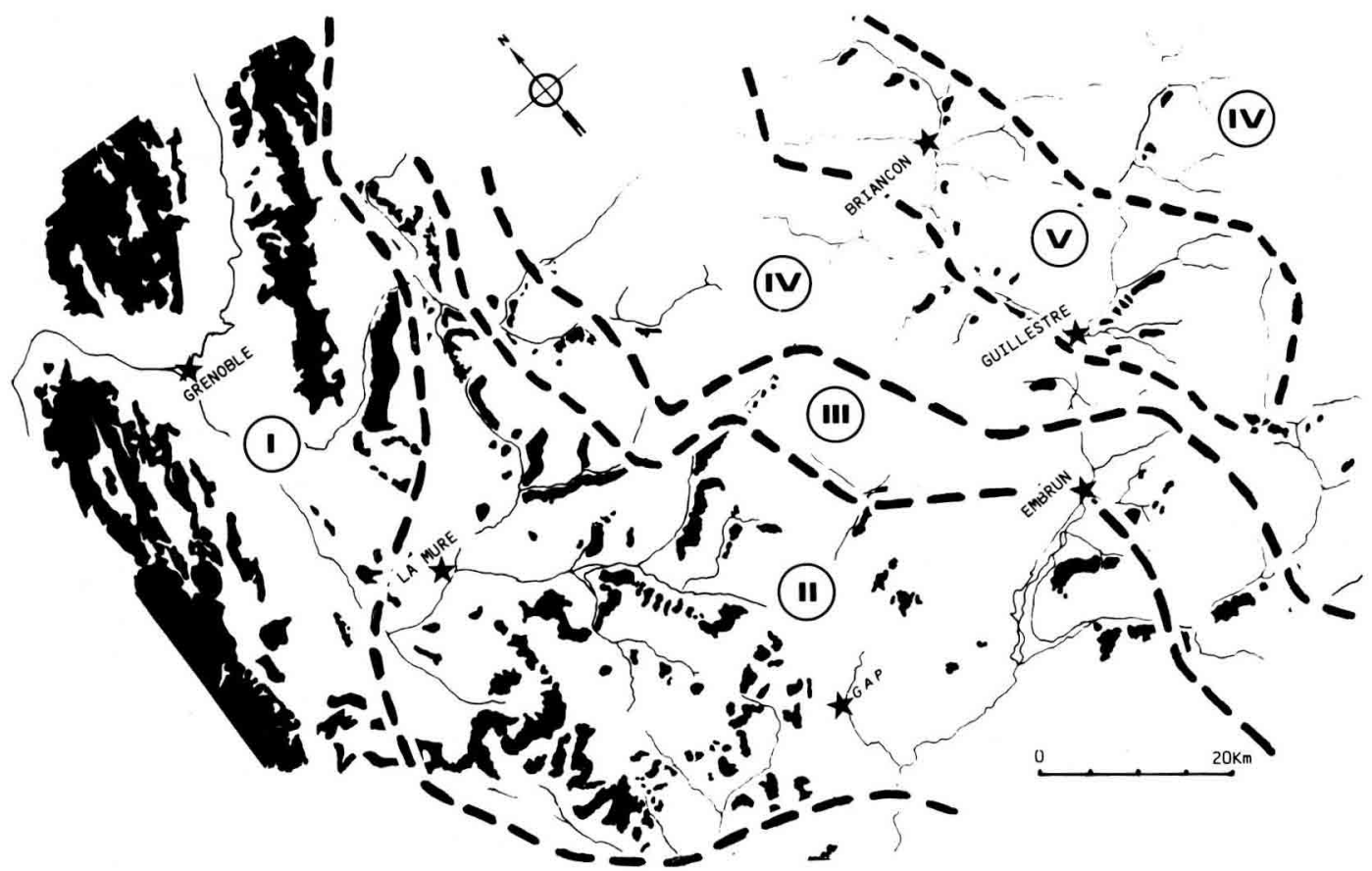

Fig 2. Localisation des principales hêtraies-sapinières et sapinières-pessières du transect étudié; divisions phytogéographiques proposées pour les Alpes dauphinoises.

sif des Ecrins; on peut aussi les subdiviser en 2 sous-secteurs.

\section{Sous-secteur ouest}

II correspond à la Haute-Romanche et au Vénéon. Le climax des adrets est difficile à imaginer à cause du fort déboisement actuel. En ubac, pessières et mélézeins constituent la totalité des forêts en raison de la disparition totale du hêtre et du sapin. Les espèces des Fagetalia sylvaticae, sont encore nombreuses. Les espèces des Betulo-Adenostyletea sont bien représentées dans les mélézeins à hautes herbes.

\section{Sous-secteur est}

C'est la partie la plus xérique qui correspond au Briançonnais (Cadel et Gilot, 1963). Dans le montagnard, les adrets sont occupés par des pinèdes sylvestres, plus rarement par des pinèdes à crochets. Les ubacs sont également le domaine des pinèdes mais aussi des mélézeins (Duchaufour et Fourchy, 1952; Ozenda et Cadel, 1981); les essences sociales externes sont absentes (hêtre) ou rares (sapin, épicéa). Dans le subalpin d'adret, le pin à crochets règne en maître. En ubac, le pin à crochets, le pin cembro, le mélèze, et plus rarement le sapin, se partagent l'espace; les groupements ont une 
tonalité assez sèche car les espèces de mégaphorbiaie sont absentes.

L'objectif de la présente étude est de tester la validité du découpage proposé par une analyse quantitative de données floristiques et écologiques. Dans cette étude, nous avons pris comme descripteurs les sapinières et les pessières. Ce choix se justifie par le fait que ces groupements sont présents, presque sans discontinuité, depuis la Chartreuse et le Vercors jusqu'en Briançonnais. Mais ce choix est bien sûr restrictif et ne saurait à lui seul caractériser chaque zone dans sa globalité; il permet, néanmoins, d'observer le long du transect étudié, la modification d'un écosystème forestier clef pour la compréhension de la végétation des Alpes dauphinoises.

Nous nous proposons de répondre aux questions suivantes :

- le découpage proposé résistera-t-il à une analyse quantitative reposant sur un échantillonnage phytoécologique homogène effectué le long d'un transect partant de la Chartreuse, pôle d'humidité des Préalpes et se terminant dans le Briançonnais, pôle de sécheresse des Alpes internes?

- Le passage d'un secteur en position externe à un secteur plus continental se traduit-il par des changements qualitatifs ou quantitatifs de la composition floristique ? Sont-ils de faible ou de forte ampleur? Interviennent-ils de façon brutale ou progressive?

- Dans le cas des sapinières et des pessières, combien de types de groupements seront validés par les analyses statistiques et quel est le degré de liaison de chacun d'entre eux avec les différentes entités phytogéographiques proposées ?

- Peut-on individualiser un lot d'espèces différentielles de chacun des secteurs et ayant, par la même, une signification sur le plan biogéographique?

- La productivité primaire des sapinières et des pessières présente-t-elle des valeurs différentes dans chacun des secteurs, si on se place dans des conditions stationnelles comparables ? Dans ce cas, les espèces individualisant les secteurs biogéographiques pourraient aussi être considérées comme des descripteurs du fonctionnement des écosystèmes de montagne.

\section{MÉTHODES}

\section{Recueil des données sur le terrain}

Deux cent soixante-quatorze relevés réunissant plus de 300 espèces constituent le support de l'information. Pour traiter cette masse de données, notamment dans son aspect quantitatif, nous avons tout d'abord fait appel à 2 méthodes de calcul multidimensionnelles couramment employées en phytoécologie : l'analyse factorielle des correspondances (AFC) et la classification ascendante hiérarchique (CAH). Une troisième méthode, l'Information mutuelle nous a apporté des précisions supplémentaires (Daget et al, 1972).

Les relevés de végétation se répartissent ainsi : - Alpes externes : Chartreuse (Bartoli, 1962; Richard, non publié); Vercors (Faure, 1968); Belledonne et Taillefer (Cadel et al, non publié; Ferrand, 1984). - Alpes intermédiaires : Oisans (Oberlinkels, 1987; Pautou, Cadel et Gillot, non publié). - Alpes internes : Massif des Ecrins (Oberlinkels, 1983; Meyer, 1981); Briançonnais et Queyras (Cadel, non publié).

Les relevés ont été effectués en ubac dans les étages montagnard et subalpin inférieur entre 640 et $2060 \mathrm{~m}$.

Le corps de données comporte des relevés sur roche mère acide et sur roche mère carbonatée. Dans la pratique, un échantillonnage systématique n'a pu être totalement réalisé dans les zones où les roches calcaires sont peu re- 
présentées (cas des Alpes intermédiaires Ouest) et dans celles où les forêts sont rares ou absentes (cas des Alpes internes Ouest). En définitive, chaque entité biogéographique est représentée par le nombre de relevés suivants : Alpes externes : 59; Alpes intermédiaires Ouest : 77; Alpes intermédiaires Est : 72; Alpes internes Ouest : 23; Alpes internes Est : 43.

Les disproportions qui existent entre ces chiffres sont donc le reflet de la variabilité des surfaces occupées par les sapinières dans chaque zone (fig 2).

Chaque relevé est accompagné d'informations topographiques et géographiques : altitude, pente, exposition, type d'humus, rochemère, coordonnées Lambert III.

\section{Traitement des données}

\section{Analyse factorielle des correspondances}

L'AFC porte sur une table de contingence, c'està-dire un tableau rectangulaire de nombres dont les sommes ont un sens tant en lignes qu'en colonnes. Dans notre cas, les 2 entrées en sont, respectivement, les espèces végétales et les relevés de végétation. Chaque élément du tableau à étudier n'est autre que la proportion moyenne du recouvrement d'une espèce dans un relevé effectué selon la méthode définie par Braun-Blanquet.

Un relevé est représenté par 1 point dans un espace comprenant autant de dimensions que d'espèces. L'AFC met en évidence des directions privilégiées de cet espace. Par projection sur les plans qu'elles définissent, on obtient une représentation du nuage de points originels. La méthode permet de représenter également les projections des espèces.

\section{Classification ascendante hiérarchique}

On remarquera que, dans la table de contingence, les lignes comme les colonnes représentent des histogrammes de fréquences relatives correspondant à des modalités qualitatives. La grandeur statistique du $\chi^{2}$ convient pour mesurer la distance qui sépare 2 lignes ou 2 coIonnes : 2 relevés ou 2 espèces. Le programme de l'ADDAD* (Fénélon, 1982), que nous avons utilisé, procède par l'agrégation successive d'éléments qui sont les plus proches. On obtient ainsi une arborescence graphique. La classification part d'éléments parfaitement individualisés et finit par les rassembler tous. En effectuant des coupures dans l'arborescence, on individualise des classes pertinentes et on établit des correspondances avec la représentation fournie par I'AFC.

\section{Information mutuelle et profil écologique indicé}

Les 2 méthodes d'analyse multidimensionnelle ont conduit à une première classification des relevés et des espèces. Le but principal de notre étude étant de quantifier les modifications du couvert végétal au sein des sapinières d'ouest en est, nous avons effectué une troisième analyse statistique à l'aide de la méthode de l'information mutuelle. Cette dernière avait pour objet de préciser la répartition quantitative des principales espèces herbacées et ligneuses le long du transect.

L'information mutuelle est une méthode statistique analytique qui permet d'étudier l'attraction des espèces vis-à-vis d'une variable écologique (Daget et al, 1972). Elle traite une table de contingence qui croise les espèces avec les modalités de la variable considérée.

L'information mutuelle est faible lorsqu'une espèce est presque toujours présente ou presque toujours absente, quelles que soient les modalités adoptées par la variable. Elle est au contraire élevée lorsqu'elle manifeste une préférence ou une répulsion pour certaines modalités de la variable. II est ainsi possible d'individualiser les espèces dont l'écologie est bien affirmée.

Le profil écologique indicé de chaque espèce complète les apports de l'information mutuelle en testant leurs sensibilités respectives à chacune des modalités de la variable écologique (Gauthier et al, 1977). L'inconvénient de cette méthode réside dans le fait que seul le caractère présence-absence est pris en considération

\footnotetext{
- ADDAD : Association pour le développement et la diffusion de l'analyse des données, 22, rue Charcot, 75013 Paris, France
} 
et non plus les abondances comme dans l'AFC et la $\mathrm{CAH}$. Nous avons utilisé les programmes mis au point par le CEPE**. Nous n'avons retenu que les 130 espèces les mieux représentées sur le transect. Certaines espèces typiquement externes mais ne figurant que dans quelques relevés ont été exclues.

La validité de ce choix a été testée par une nouvelle AFC qui a porté sur les 274 relevés et les 130 espèces considérées (tableau II).

\section{RÉSULTATS}

\section{Commentaire des plans factoriels} (fig 3 et 4)

Nous nous bornerons à la description du plan 1-2 qui se prête le mieux à une inter- prétation phytoécologique car les espèces s'y disposent selon un étalement maximum et font apparaitre des gradients significatifs.

Les 4 premiers axes expliquent $13 \%$ de la variation totale; cette valeur est faible mais courante dans les études phytoécologiques (Lacoste, 1972).

\section{Axe 1}

Cet axe répartit les espèces selon un gradient, reflet du niveau d'activité biologique de l'humus. Les espèces les mieux corrélées positivement le long de cet axe sont : Acer pseudoplatanus, Adenostyles alliariae, Cicerbita alpina, Galium odoratum; toutes, sauf la dernière, sont des mésohygrophiles de mull.
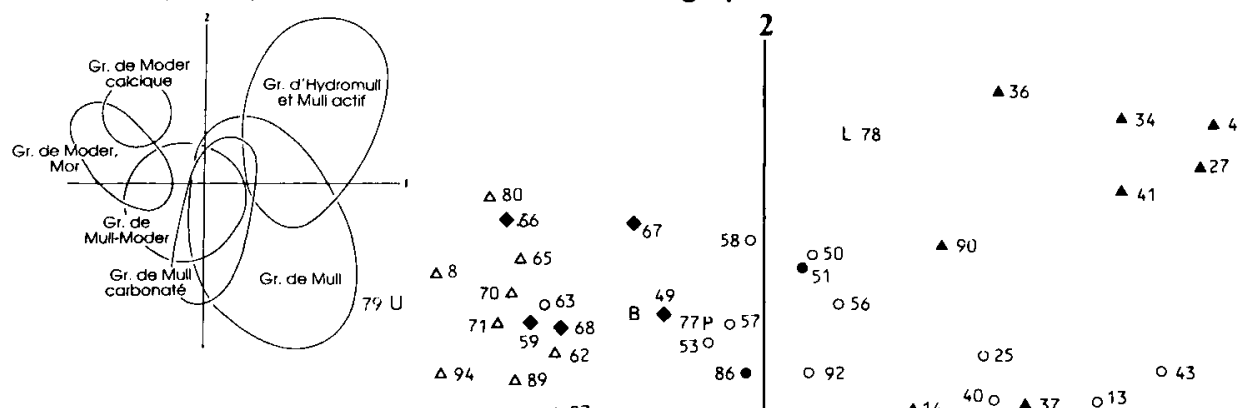

$77 P \circ 57$
530
$86 \bullet$

056

$092025 \quad 043$

$\begin{array}{rrr}-35 & 4140037 & 013 \\ 400025 & \Delta 29\end{array}$

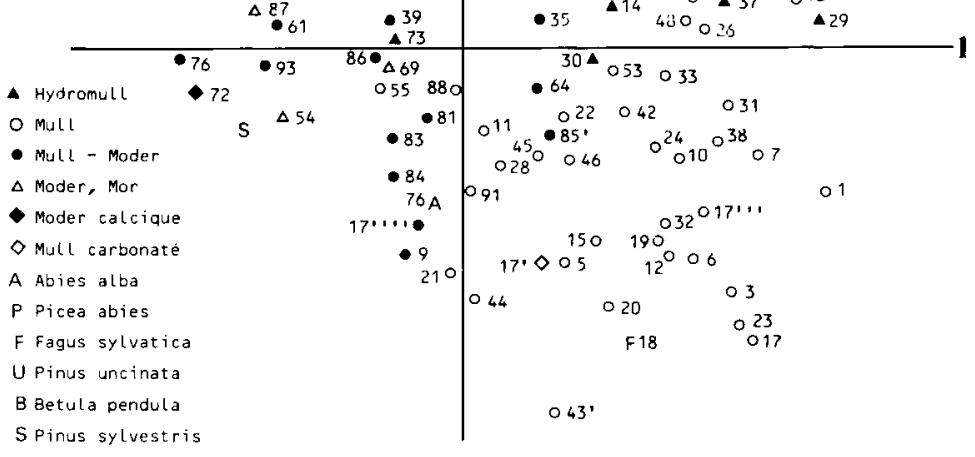

Fig 3. Position des espèces projetées sur le plan factoriel 1-2 selon l'appartenance aux groupes écologiques.

** CEPE : Centre d'études phytosociologiques et écologiques, Montpellier, France. 


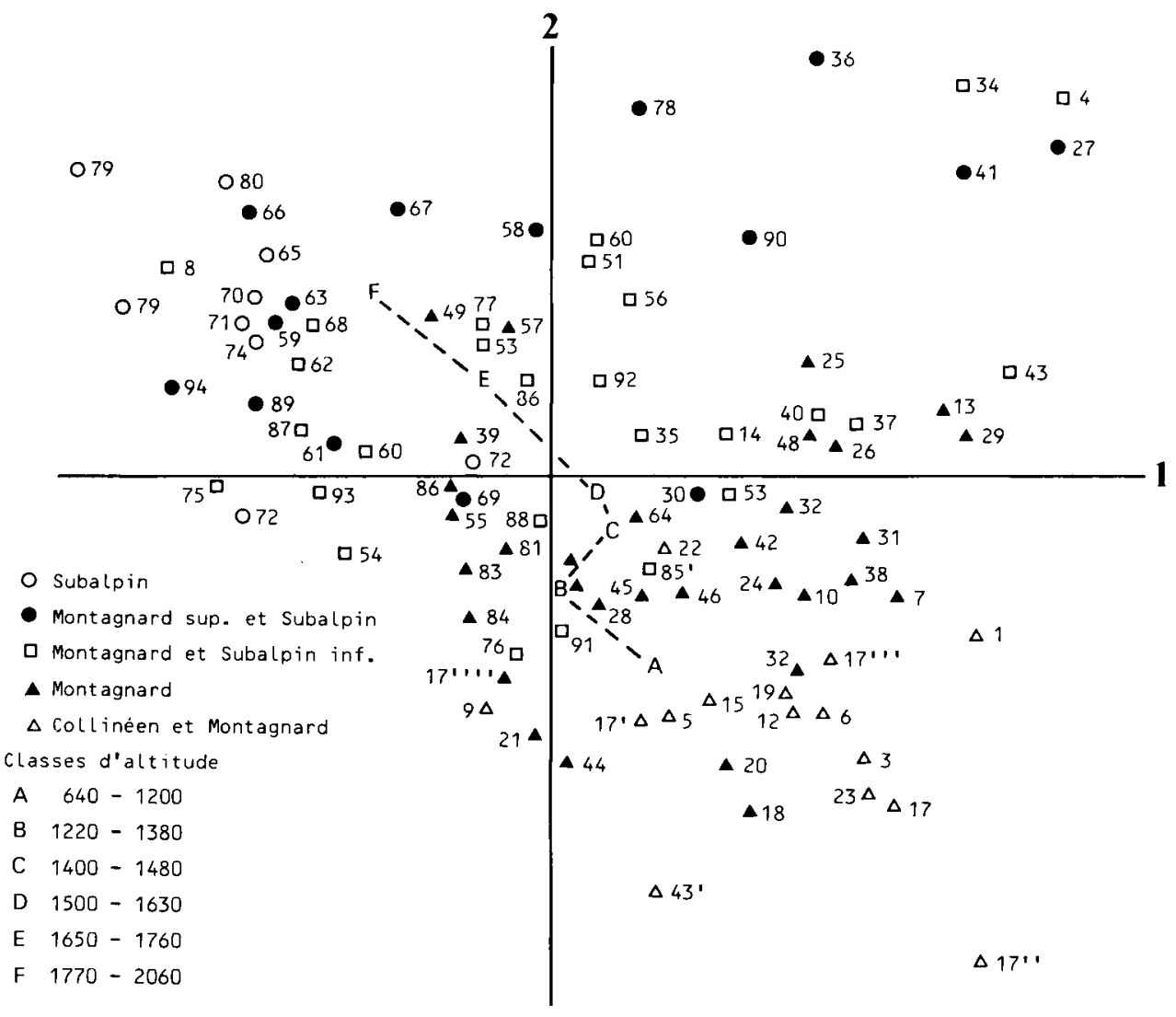

Fig 4. Position des espèces projetées sur le plan factoriel 1-2 selon les étages de végétation et projection des classes d'altitude (variable supplémentaire).

Du côté négatif figurent : Melampyrum sylvaticum, Vaccinium myrtillus, $V$ vitisidaea, Homogyne alpina, Festuca flavescens; il s'agit là d'espèces mésophiles de moder.

\section{Axe 2}

Les espèces se distribuent selon un gradient altitudinal assez net.

Du côté positif figurent : Larix decidua, Viola biflora, Picea abies, Rumex arifolius, Adenostyles alliariae. Du côté négatif s'in- dividualisent: Abies alba, Fagus sylvatica, Galium odoratum, Festuca sy/vatica.

\section{Axe 3}

Les espèces sont distribuées selon une orientation à peu près nord-sud, opposant les espèces des Préalpes du Nord, sciaphiles et plus ou moins acidophiles du montagnard aux espèces héliophiles les plus méridionales et caractéristiques de milieux plus ouverts. 


\section{Axe 4}

L'axe 4 oppose les espèces caractéristiques des sapinières typiques à celles qui sont les plus éloignées de la sapinière sensu stricto et qui s'épanouissent dans les groupements de basse altitude.

Les relevés appartenant aux Alpes externes et aux Alpes intermédiaires sont dispersés dans l'ensemble du plan; ce type de distribution s'explique par la très grande diversité des groupements se rattachant au complexe de la hêtraie-sapinière sous un climat frais et bien arrosé. C'est la grande diversité des substrats depuis les calcaires durs urgoniens jusqu'aux amphibolites qui explique le grand nombre de groupements décrits (Bartoli, 1962; Faure, 1968; Ferrand, 1984).

\section{Les résultats de la CAH (tableau l et fig 5)}

Deux grandes unités apparaissent, d'une part les hêtraies-sapinières qui rassem-

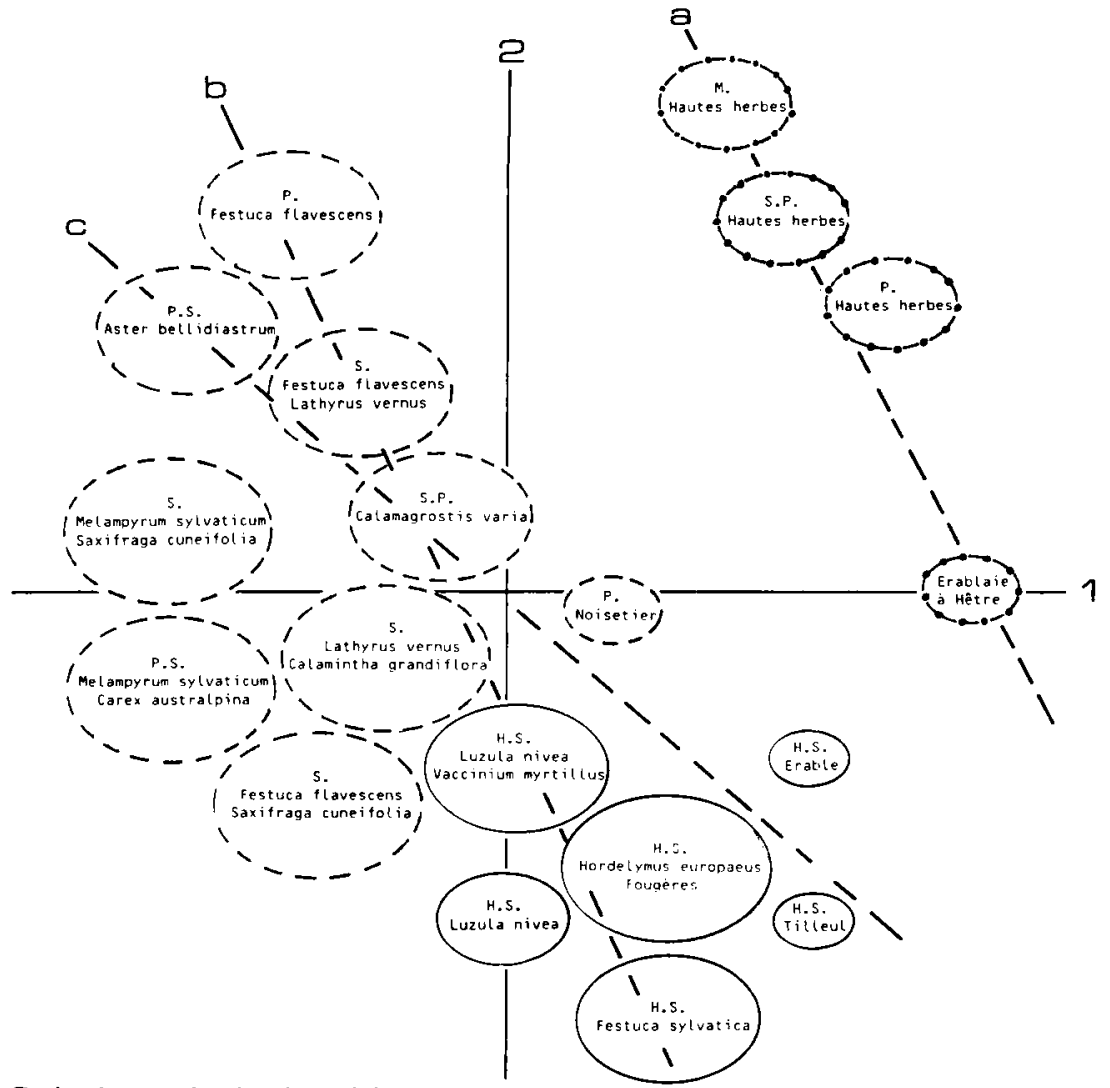

Fig 5. Projection sur le plan factoriel 1-2 des groupements végétaux obtenus par la CAH et matérialisation de directions privilégiées $(a, b, c)$. _ : groupements des Alpes externes et intermédiaires occidentales; - - - - : : groupements des Alpes internes et intermédiaires orientales; $\longrightarrow$ - - : groupements à hautes herbes (Alpes externes et intermédiaires). $\mathrm{S}:$ sapinière; $\mathrm{P}$ : pessière; $\mathrm{H}$ : hêtraie; $M$ : mélézein. 


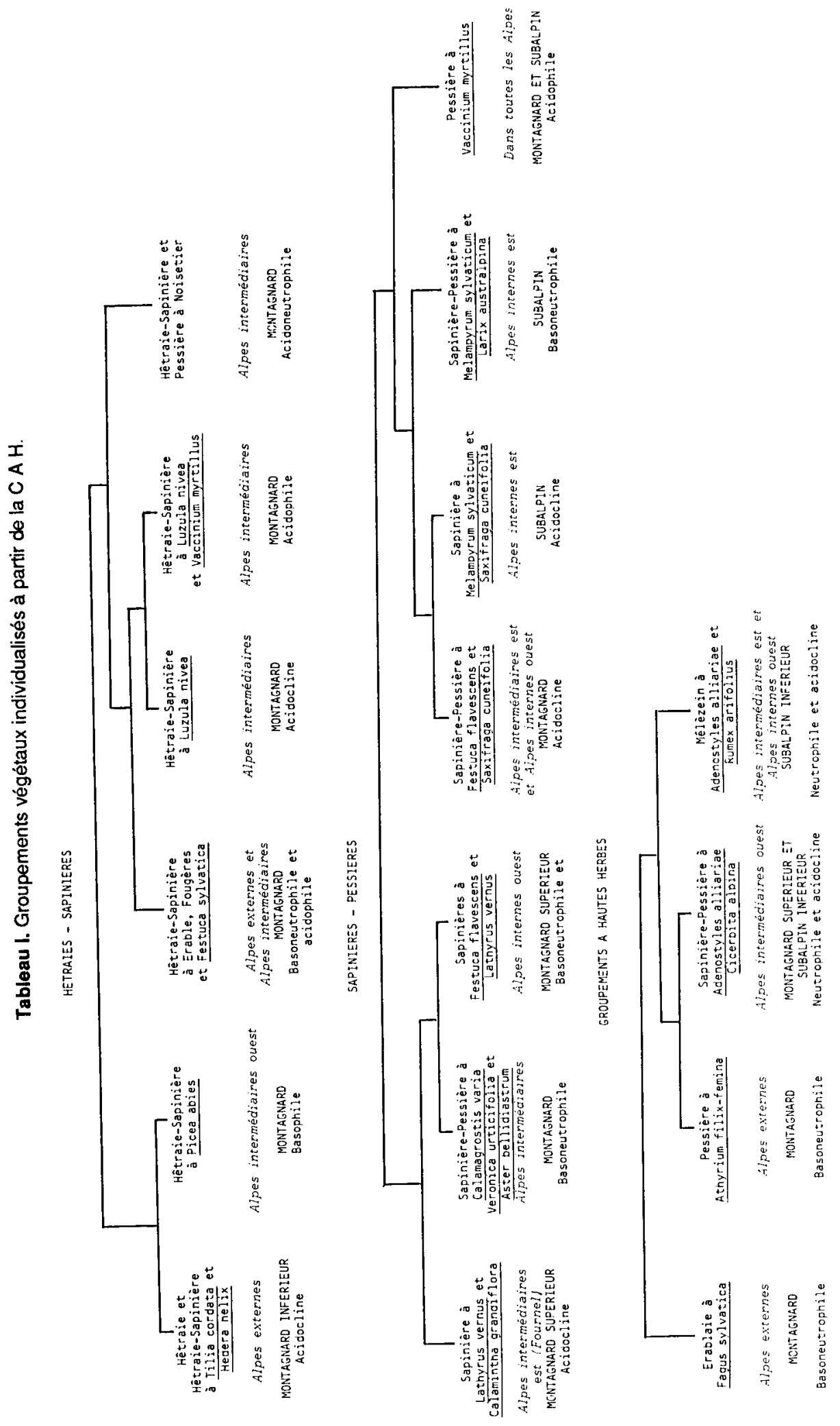


blent les groupements forestiers des Alpes externes et intermédiaires ouest, et d'autre part, les sapinières-pessières des Alpes intermédiaires orientales et des Alpes internes. Chacune de ces 2 grandes unités est subdivisée en groupements floristiquement, écologiquement et biogéographiquement définis (tableau 1). Une troisième unité comprend les groupements à hautes herbes (niveaux I à IV) riches en espèces mésohygrophiles de mull (mégaphorbiaies).

\section{Direction a}

Les groupements riches en espèces mésohygrophiles de mull se distribuent le long d'un gradient altitudinal. Ce sont les groupements présents depuis les Alpes externes (érablaie à hêtre, pessière à Athyrium filix-femina) jusqu'aux Alpes internes occidentales (sapinière-pessière et mélézein à Adenostyles alliariae). Le soussecteur interne est ne présente pas ce type de groupements au sein des sapinières.

\section{Direction b}

Elle met en lumière le passage des hêtraies-sapinières riches en hêtre aux pessières pures par l'intermédiaire des sapinières et des sapinières-pessières. Méritent une mention particulière les sapinières caractéristiques des Alpes intermédiaires orientales comme celle du Fournel (Briançonnais occidental) (Meyer, 1981) ou des Alpes internes occidentales comme celle de Marassan (Queyras oriental) dans lesquelles le hêtre et l'épicéa sont absents, mais où le mélèze est fréquent. La sapinière de Marassan à Festuca flavescens et Lathyrus vernus est située sur schistes lustrés où quelques espèces calciphiles comme Aster bellidiastrum sont présentes; c'est sans doute la raison qui explique la position un peu particulière de ce groupement. Le caractère transitoire de la pessière à noisetier se retrouve dans la position qu'elle occupe sur le graphique; elle se situe à proximité des hêtraiessapinières avec lesquelles elle partage fraîcheur et richesse chimique du sol (Oberlinkels, 1987).

\section{Direction c}

Les groupements uniquement présents sur calcaire s'ordonnent le long de cette direction : hêtraies-sapinières calciphiles des zones externe et intermédiaire occidentales, sapinière-pessière à Calamagrostis varia et à Veronica urticifolia du soussecteur intermédiaire occidental, pessièresapinière à Aster bellidiastrum du soussecteur intermédiaire oriental (Pautou et al, 1986). La sapinière-pessière à Melampyrum sylvaticum et à Carex australpina caractéristique du sous-secteur interne oriental, sur calcaire, s'apparente beaucoup plus à la sapinière à Melampyrum sylvaticum et à Saxifraga cuneifolia sur roche mère acide du même sous-secteur plutôt qu'à la sapinière-pessière calciphile à Aster bellidiastrum précitée. Dans le Briançonnais, les sapinières ont une composition floristique très particulière où dominent les espèces acidophiles de moder à peine désaturé; la différence de substrat se traduit par l'absence ou la présence de quelques calciphiles, dont Carex australpina est la plus caractéristique.

L'AFC et la $\mathrm{CAH}$ individualisent de façon très nette des relevés des Alpes internes. En revanche, les relevés appartenant aux Alpes externes et aux Alpes intermédiaires sont dispersés sur l'ensemble du plan. Le tableau II, construit à partir de l'information mutuelle, permet d'aller audelà d'une partition en deux entités. 


\section{Apport de l'information mutuelle et analyse des profils écologiques indicés (tableau II et fig 6)}

Le tableau met en lumière de façon très nette qu'un fort contingent d'espèces est attiré par les conditions écologiques des secteurs qui occupent une position marginale; ainsi, 16 espèces présentent une forte attirance pour le niveau I, dont 13 de façon très significative; 18 espèces présentent une forte attirance pour le niveau $\mathrm{V}$, dont 14 de façon très significative (de 63 jusqu'à 75). On peut expliquer cette
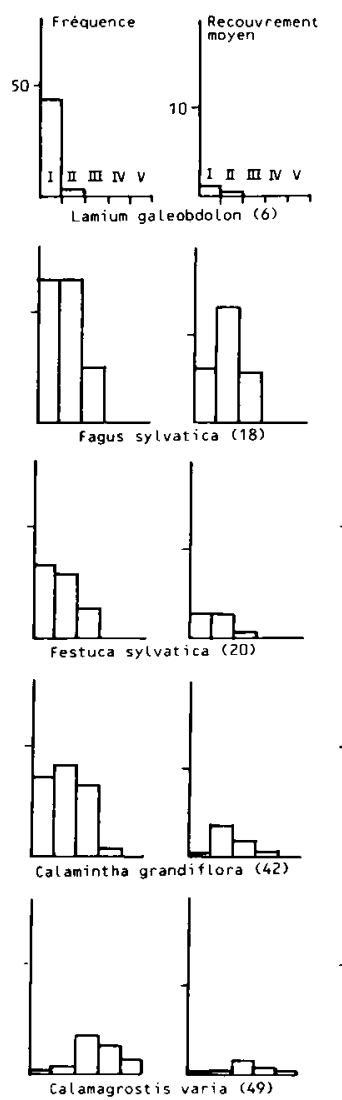
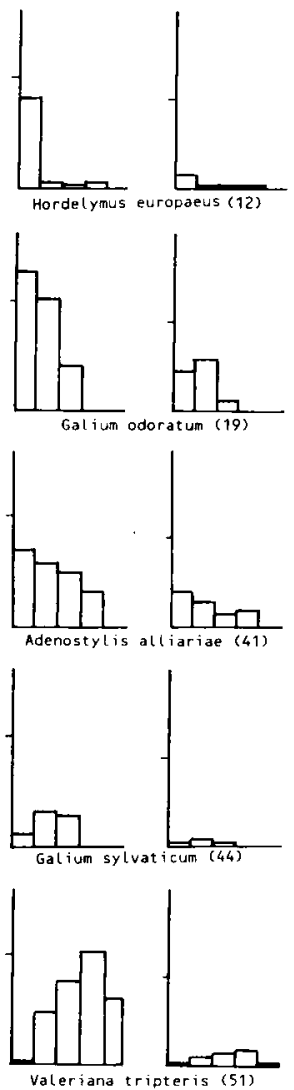

forte attraction des pôles extrêmes par des causes de type chorologique (origine des taxons) et de type écologique (caractéristiques climatiques affirmées).

Cinq espèces sont attirées par les niveaux I et II dont 2 de façon très significative; c'est le cas de Fagus sylvatica. Par un même effet de symétrie, 5 espèces sont attirées par les niveaux $V$ et IV, dont 2 façon très nette; c'est le cas de Festuca flavescens, espèce qui présente un fort recouvrement dans la partie occidentale des Alpes internes. La distribution de ces groupes d'espèces semble être régie par
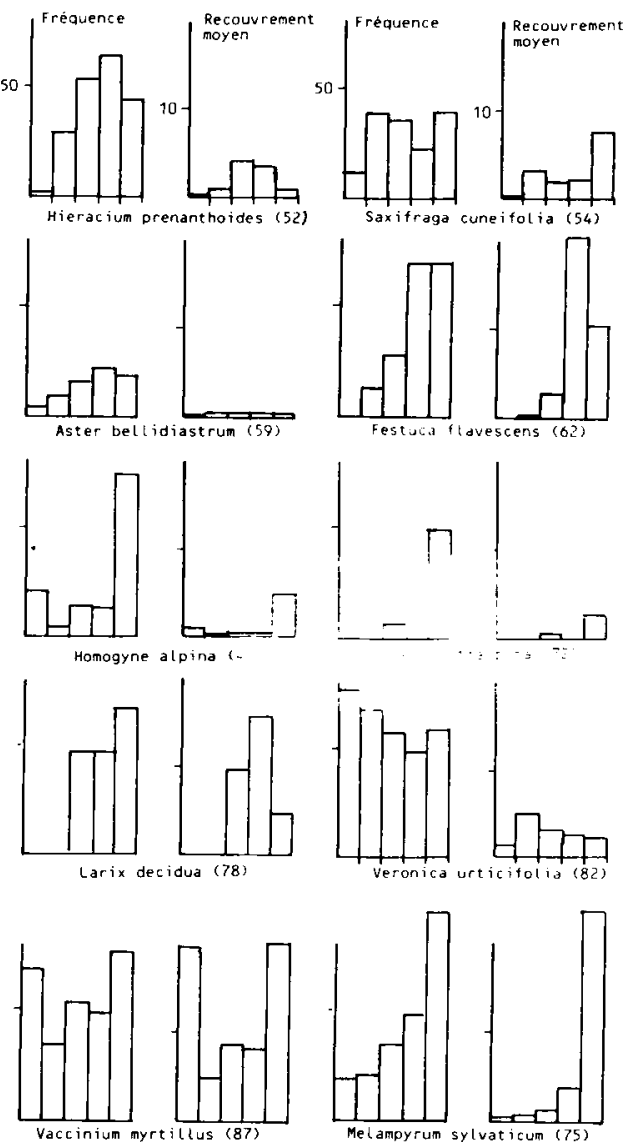

Fig 6. Contribution en pourcentages des différentes espèces retenues dans les 5 niveaux biogéographiques proposés, exprimée par la fréquence (coefficients de présence-absence) et par le recouvrement moyen. 
Tableau II. Affinités des espèces vis-à-vis des 5 niveaux biogéographiques retenus, exprimées par le profil indice. Les espèces sont classées par ordre d'affinité progressive vis-à-vis des 5 niveaux biogéographiques. La sensibilité de chaque espèce à l'égard de chacun des niveaux s'exprime par le profil écologique indicé. L'absence de signe indique l'insuffisance de données pour tester la sensibilité de l'espèce par rapport au niveau considéré.

\begin{tabular}{|c|c|c|c|c|c|c|}
\hline \multicolumn{2}{|r|}{ NOMS DES ESPECES } & \multirow{2}{*}{$\frac{\mathrm{I}}{++}$} & \multirow[t]{2}{*}{11} & \multirow[t]{2}{*}{111} & \multirow[t]{2}{*}{ IV } & \multirow[t]{2}{*}{ v } \\
\hline$?$ & Impatiens noli-tangere & & & & & \\
\hline 2 & Asplenium viride & +++ & & & & \\
\hline 3 & Circaea lutetiana & +++ & & & & \\
\hline 4 & Lysimachia nemorum & +++ & & - & & \\
\hline 5 & Carex sylvatica & +++ & -- & -- & & - \\
\hline 8 & Lamium galeobdolon & +++ & - & --- & & - \\
\hline 7 & Valeriana officinalis & +++ & & & & \\
\hline 8 & Blechnum spicant & +++ & & - & & \\
\hline 9 & Ilex aquifolium & +++ & & & & \\
\hline 10 & Bromus asper & +++ & & & & \\
\hline 11 & Cardamine heptaphyila & +++ & - & - & & \\
\hline 12 & Hordelymus europaeus & +++ & -- & - & & $=$ \\
\hline 13 & Athyrium fjlix-femina & +++ & $\cdot$ & -- & & - \\
\hline 74 & Polygonatum verticillatum & +++ & . & -- & & --- \\
\hline 15 & Saricula eur opaea & + & - & & & \\
\hline 16 & Streptopus amplexifolius & + & . & & & \\
\hline 17 & Ulmus glabra & $\cdot$ & $\cdot$ & & & \\
\hline 18 & Fagus sylvatica & +++ & +++ & -- & --- & ... \\
\hline 19 & Galium odoratum & +++ & +++ & -- & $\cdots$ & $\cdots$ \\
\hline 20 & Festuca syivatica & +t & + & . & & - \\
\hline $2 ?$ & Galium rotundifolium & +++ & + & - & --- & -- \\
\hline 22 & Ajuga reptans & +++ & . & . & $+\infty$ & --- \\
\hline 23 & Mercurialis perennis & $\cdot$ & . & - & & \\
\hline 24 & Polystichum aculeatum & +++ & . & - & & +- \\
\hline 25 & Epilobium montanum & + & - & . & - & $\cdots$ \\
\hline 26 & Milium effusum & + & - & - & - & \\
\hline 27 & Cicerbita alpina & + & . & $\cdot$ & - & \\
\hline 28 & Neottia nidus-avis & + & . & - & & - \\
\hline 29 & Stellaria nemorum & + & . & - & & - \\
\hline 30 & Thalictrum aquilegifolium & + & . & . & & - \\
\hline 37 & Actaea spicata & & & . & & -- \\
\hline 32 & Aruncus dioicus & . & . & . & & -- \\
\hline 33 & Geranium robertianum & ++ & . & . & & -- \\
\hline 34 & Saxifraga rotundifolia & + & , & . & & - \\
\hline 35 & Gymnocarpium dryopteris & . & . & . & & \\
\hline 36 & Myosotis sylvatica & . & . & . & & - \\
\hline 37 & Ranunculus aconitifolius & ++ & ++ & . & + & --- \\
\hline 38 & Dryopteris filix-mas & +++ & . & . & . &.- \\
\hline 39 & Melampyrum nemorosum & + & . & . & . & -- \\
\hline 40 & Senecio fuchsil & . & . & . & . & --- \\
\hline 41 & Adenostyles alliariae & - & . & . & . & $\cdots$ \\
\hline 42 & Calamintha grandiflora & . & t+ & . & -- & --- \\
\hline 43 & Acer pseudoplatanus & . & + & . & -- & --- \\
\hline 44 & Galium sylvatıcum & & + & . & & - \\
\hline 45 & Mycel is mural is & . & . & + & & -- \\
\hline 46 & Paris quadrifolia & . & . & + & - & $\cdots$ \\
\hline \multirow[t]{6}{*}{47} & Adenostyles alpina & - & - & + & & \\
\hline & Degré de sensibilité & tirance & & ulsion & & \\
\hline & Yes sigrificatide & $+*$ & & -- & & \\
\hline & $\sin \sin ^{\circ} a^{+}=$ & +. & & $\cdots$ & & \\
\hline & 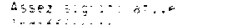 & + & & - & & \\
\hline & $\therefore-6 a^{2}+2 \cdots=$ & & & . & & \\
\hline
\end{tabular}

les mêmes causes que dans le cas des catégories précédentes, mais il s'agit d'espèces ayant une plus grande amplitude écologique.

Le nombre d'espèces attirées de façon significative par les niveaux médians est nettement plus bas : 4 par le niveau II (dont Abies alba), 6 par le niveau III, 1 par le niveau IV. Le fait qu'il n'y ait pas d'espèces attirées de façon significative par les niveaux II et III montre que les Alpes intermédiaires constituent une interface entre 2 contingents floristiques orientaux et occidentaux comportant des espèces à écolo-

\begin{tabular}{|c|c|c|c|c|c|c|}
\hline \multicolumn{2}{|c|}{ NOMS DES ESPECES } & I & Il & 111 & IV & $v$ \\
\hline $\begin{array}{l}48 \\
49\end{array}$ & $\begin{array}{l}\text { Aegopodium podagraria } \\
\text { Calamagrostis varia }\end{array}$ & - & & $\begin{array}{c}+++ \\
++\end{array}$ & & \\
\hline 50 & Melica nutans & -- & . & + & & - \\
\hline 51 & Valeriana tripteris & --- & . & + & + & . \\
\hline $\begin{array}{l}52 \\
53\end{array}$ & Hieracium prenanthoides & -- & . & +++ & ++ & \\
\hline 54 & $\begin{array}{l}\text { Lonicera alpigena } \\
\text { Saxifraga cuneifolia }\end{array}$ & - & - & $\cdot$ & : & - \\
\hline 55 & $\begin{array}{l}\text { Saxifraga cuneisolia } \\
\text { Lathyrus vernus }\end{array}$ & $\overline{---}$ & & : & : & $:$ \\
\hline & Chaerophyllum villarsia & & $\cdots$ & & +t+ & \\
\hline & Ranunculus nemorosus & --- & - & ++ & : & + \\
\hline 58 & Geranium sylvaticum & $\begin{array}{l}- \\
-\end{array}$ & $=$ & $:$ & $\stackrel{+}{+}$ & $\stackrel{++}{+}$ \\
\hline 59 & $\begin{array}{l}\text { Aster bellidiastrum } \\
\text { Hepatica nobilis }\end{array}$ & $\ldots$ & - & : & + & $\stackrel{+}{++\infty}$ \\
\hline $\begin{array}{l}60 \\
67\end{array}$ & $\begin{array}{l}\text { Hepatica nobilis } \\
\text { clematis alpina }\end{array}$ & -- & -- & : & + & $\begin{array}{l}t++t \\
++t\end{array}$ \\
\hline 62 & Festuca flavescens & --- & --- & $:$ & +++ & t+m \\
\hline 63 & Gentiana lutea & --- & . & : & . & ++ \\
\hline 64 & orthilia secunda & & . & - & : & +++ \\
\hline 65 & Soldanella alpina & -- & - & 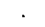 & & +++ \\
\hline 66 & Sesleria albicans & $\cdots$ & - & . & & +++ \\
\hline 67 & Valeriana montana & - & - & . & & t++ \\
\hline & Polygala chamaebuxus & & -- & . & & $+t+$ \\
\hline 69 & $\begin{array}{l}\text { Sorbus chamaemespi lus } \\
\text { pan }\end{array}$ & . & - & . & . & +++ \\
\hline 71 & $\begin{array}{l}\text { Rhododendron ferrugineum } \\
\text { Homogyne alpina }\end{array}$ & - & - & . & . & ++ \\
\hline 72 & $\begin{array}{l}\text { Homogyne alpina } \\
\text { Carex australpina }\end{array}$ & $\therefore$ & $\cdots$ & $\therefore$ & & tit \\
\hline 73 & $\begin{array}{l}\text { Parex dustrapipina } \\
\text { Phyteuma ovatum }\end{array}$ & $\overline{-}$ & $\cdots$ & : & . & $\stackrel{+++}{+++}$ \\
\hline 74 & Pulsatilla alpina & -- & -- & : & : & +++ \\
\hline & Melampyrum sylvaticum & --- & -- & . & . & +t+ \\
\hline $\begin{array}{l}76 \\
77\end{array}$ & Abies alba & $\therefore$ & +++ & - & --- & \\
\hline 78 & $\begin{array}{l}\text { 1cead ables } \\
\text { Larix decidua }\end{array}$ & +++ & $\therefore$ & & . & $\cdots$ \\
\hline & $\begin{array}{l}\text { Larix decidua } \\
\text { Pinus uncinata }\end{array}$ & - & - & + & . & $\stackrel{+++}{+++}$ \\
\hline 80 & Pinus cembra & & & & & + \\
\hline & Prenanthes purpurea & +++ & . & - & -- & . \\
\hline 82 & Veronica urticifolia & + & . & . & . & it \\
\hline & $\begin{array}{l}\text { Luzula nivea } \\
\text { Hieracium murorum }\end{array}$ & & : & : & : & $\begin{array}{l}++ \\
+++\end{array}$ \\
\hline $\begin{array}{l}84 \\
85\end{array}-20$ & $\begin{array}{l}\text { Hieracium murorum } \\
\text { Rosa penduliria }\end{array}$ & +4 & $\therefore$ & :- & : & ++ \\
\hline 86 & $\begin{array}{l}\text { Rosa pendulina } \\
\text { Luzula sylvatica }\end{array}$ & 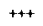 & --- & . & . & $++t$ \\
\hline & Vaccinium myrtillus & + & -- & & & ++ \\
\hline $\begin{array}{l}88 \\
89\end{array}$ & $\begin{array}{l}\text { Euphorbia dulcis } \\
\text { Luzula luzulina }\end{array}$ & -- & $\stackrel{+}{--}$ & $:$ & $\because$ & t+ \\
\hline 90 & $\begin{array}{l}\text { Luzula lunuina } \\
\text { Peucedanum ostruthium }\end{array}$ & & . & . & & : \\
\hline 91 & Phyteuma spicatum & . & . & . & -- & . \\
\hline 92 & $\begin{array}{l}\text { Sorbus aucuparia } \\
\text { Oaj }\end{array}$ & & & : & : & $\therefore$ \\
\hline $\begin{array}{l}93 \\
94\end{array}$ & $\begin{array}{l}\text { Oxal is acetosella } \\
\text { Vaccinium vitis-idaea }\end{array}$ & ++ & $\therefore$ & $\therefore$ & $\cdot$ & $\mathrm{H+}$ \\
\hline
\end{tabular}

gie affirmée. Deux cas particuliers sont à signaler : celui des espèces attirées par les niveaux III et IV, c'est-à-dire trouvant leur optimum au niveau de l'écotone Alpes intermédiaires-Alpes internes; celui des espèces à répartition discontinue, qui sont le reflet de caractéristiques pédologiques très marquées; ainsi, le groupe des espèces acidophiles strictes comme Vaccinium myrtillus. La répartition de cette espèce qui est attirée par les 2 niveaux extrêmes est révélateur d'un phénomène de discontinuité induit par la nature du substrat, au sein de la variation progressive d'ordre climatique 
d'ouest en est. En zone externe, la myrtille est bien représentée sur les schistes cristallins de Belledonne et sur le calcaire urgonien de Chartreuse ici recourvert d'humus brut. Dans la partie orientale des Alpes internes, l'altitude élevée et la fréquence des moder favorisent également cette espèce. Par contre, les sols de la zone intermédiaire essentiellement formés sur amphibolite, sur schiste ou sur calcaire marneux, présentent une forte activité biologique et constituent donc un obstacle pour une espèce acidophile.

Les espèces figurant en fin de tableau présentent une répartition biogéographique hétérogène, bien qu'étant globablement plus attirées par le niveau $V$ (Alpes internes orientales). II s'agit là, pour la plupart, d'espèces acidoclines et acidophiles fréquentes dans toutes les sapinières d'altitude du sous-secteur interne oriental.

\section{DISCUSSION}

Les analyses statistiques portant sur les groupements ou sur les espèces soulignent la forte individualisation des niveaux extrêmes.

\section{Caractéristiques des différents secteurs phytogéographiques}

\section{Alpes externes}

Le secteur constitue une entité homogène sur le plan phytogéographique et sociologique. Ce n'est pas étonnant quand on constate que le total des précipitations varie faiblement depuis les massifs préalpins jusqu'à la crête de Belledonne. La vallée du Grésivaudan ne peut être retenue comme limite entre Alpes externes et Alpes intermédiaires (Cadel et Pautou,
1984; Ozenda, 1985). Certes, il existe des différences très nettes entre les massifs préalpins calcaires et la chaîne de Belledonne où dominent les amphibolites mais, contrairement à ce que l'on pourrait croire, les différences de nature chimique de la roche mère n'ont pas d'influence sur l'abondance des espèces acidophiles. Sur les massifs calcaires (urgonien), il y a formation d'un humus brut très acide; il n'est pas étonnant qu'un grand nombre d'acidophiles se rencontrent à la fois en Chartreuse, en Vercors sur calcaire et dans les massifs cristallins. Par contre la granulométrie des sols obtenus à partir de la décomposition des roches mères est à l'origine d'une capacité hydrique très variable. Dans les massifs siliceux, l'altération des amphibolites conduit à la formation de sols riches en éléments fins et en colloïdes argileux : les espèces mésohygrophiles des mégaphorbiaies sont nombreuses et ont un fort recouvrement. En revanche, les calcaires des Préalpes sont à l'origine de sols pauvres en argiles, plus filtrants d'où une diminution des espèces ayant des besoins en eaux élevés.

\section{Alpes intermédiaires}

Les analyses statistiques confirment que les Alpes intermédiaires constituent une cellule charnière, une plaque tournante qui accueille des peuplements aussi bien d'origine occidentale que d'origine orientale mais souvent appauvris en leurs éléments les plus représentatifs. Les peuplements appartenant aux trois ensembles individualisés par la $\mathrm{CAH}$ y sont représentés mais à la faveur de situations écologiques différentes (phénomène de juxtaposition).

Cette organisation en mosaïque s'explique par l'existence de discontinuités d'ordre édaphique, topographique ou orographique. La diminution considérable des précipitations d'ouest en est (environ 100 
mm par $10 \mathrm{~km}$ ) est atténuée par des facteurs de compensation d'origine édaphique et aggravée par l'existence de contraintes stationnelles sur le plan de l'économie de l'eau, susceptibles d'annihiler les changements d'ordre climatique (diminution de précipitations).

Les conséquences sur le plan de la chorologie sont nombreuses :

- les groupements à hêtre dominant (sapin et épicéa rares ou absents) parviennent à la limite orientale des Alpes intermédiaires (Vénéon) à la faveur de sols superficiels dans les stations à exposition est ou ouest. En revanche, des sapinières-pessières sont présentes dès l'entrée des Alpes intermédiaires sur des roches mères très acides conduisant à la formation d'humus de type moder;

- les sapinières se rencontrent depuis le montagnard moyen jusqu'au subalpin inférieur; à ce niveau, sapin et épicéa peuvent coexister dans des groupements mixtes. Suivant les régions, l'une ou l'autre des 2 espèces peut être absente. Ainsi, dans le bas Vénéon, le sapin est rare et c'est l'épicéa qui occupe la totalité des ubacs entre 1000 et $1800 \mathrm{~m}$ (Tonnel et Ozenda, 1964; Oberlinkels, 1983); la composition floristique de ces forêts est trèc comparable à celle que l'on rencontre dans les sapinières-pessières du Valjouffrey (fig 1). Plus au sud, le Valgaudemar, où l'épicéa est quasi absent, présente des sapinières pures dont la composition floristique s'apparente fortement à celle du Valjouffrey (Oberlinkels, 1987). Ainsi, dans les Alpes intermédiaires, l'individualisation d'une série de la sapinière-pessière s'impose (Pautou et al, 1986). Mais il s'agit, en fait, d'une unité compréhensive qui existe sous plusieurs types physionomiques. Cette hétérogénéité est le reflet de l'histoire postglaciaire (arrivée récente de Picea abies depuis 1000 ans en Oisans) (Coûteaux, 1981), des sylvicultures passées et des va- riations locales des conditions du milieu. Deux types principaux de sapinières sont représentés sur calcaire, la sapinièrepessière à Calamagrostis varia et la sapinière-pessière à Aster bellidiastrum (Pautou et al, 1986; Oberlinkels, 1987);

- la présence de calcaires marneux dans le bassin de Bourg d'Oisans favorise, tout autant que la diminution des précipitations, l'épanouissement du pin sylvestre et le maintien de hêtraies-pinèdes stables, groupements qui ont à la fois un déterminisme climatique et édaphique (Pautou et al, 1986);

- la vigueur de l'activité érosive en Oisans provoque la dénudation de sites sur de vastes superficies; ces sites sont alors très favorables au mélèze. Ces conditions ainsi que les interventions humaines (reboisements) semblent plus à l'origine de la progression des populations de mélèze que les changements d'origine climatique;

- la présence de roches mères conduisant à la formation de sols à forte activité biologique et à bonne rétention hydrique (amphibolites, calcaires marneux), l'existence de névés, expliquent la forte représentation des peuplements à hautes herbes et des espèces de mull et d'hydromull dans les Alpes intermédiaires. Le passage du sous-secteur occidental au sous-secteur oriental se manifeste par une diminution sensible des représentants des Fagetalia sylvaticae parallèlement à l'apparition d'espèces telles que Festuca flavescens, Larix decidua, Carex australpina; en outre, Calamagrostis varia, présente dans le soussecteur oriental une extension maximale (Oberlinkels, 1987) alors que dans la zone externe, il préfère le couvert modéré des pinèdes; cette forte extension peut être également reliée à l'abondance des calcaires liasiques. L'Abietetum albae festucetosum (Kuoch, 1954) bien représenté dans les Alpes intermédiaires occidentales laisse la place à l'Abietetum albae melam- 
pyretosum dans les Alpes intermédiaires orientales. En fait, les espèces qui ont un fort recouvrement dans la zone intermédiaire semblent répondre plus à des exigences d'ordre édaphique (Calamagrostis varia, Adenostyles glabra sur calcaire marneux notamment), qu'à des exigences climatiques.

\section{Alpes internes}

La partition des Alpes internes en 2 entités est corroborée par tous les tests statistiques. Les pessières à Festuca flavescens et Saxifragra cuneifolia qui s'implantent sur les fortes pentes schisteuses et siliceuses du Vénéon (Oberlinkels, 1983) et les mélézeins à mégaphorbiaie sur les sols profonds de la Haute Romanche sont représentatifs du sous-secteur occidental (niveau IV). Les sapinières à Festuca flavescens et Lathyrus vernus situées sur les schistes lustrés du Queyras oriental sont aussi à rattacher au niveau IV. II est intéressant de remarquer que la partie la plus à l'est des Alpes françaises est plus humide que le Briançonnais ou le Queyras occidental : cela est dû à l'influence des conditions climatiques règnant sur les Alpes italiennes toute proches. Dès que l'on passe la frontière, la pluviosité augmente très rapidement et l'on ne tarde pas à rencontrer à nouveau, le hêtre.

Le sous-secteur oriental s'individualise négativement par l'absence des peuplements indiqués pour les Alpes internes occidentales et positivement par l'extension massive des pinèdes sylvestres (Onobrychideto-Pinetum, Ononido-Pinetum, Deschampsio-Pinetum) (Braun-Blanquet, 1961) et des pinèdes à crochets. II constitue le noyau le plus xérique qui n'a pas d'équivalent dans tout l'arc alpin même si des régions comme le Vinschgau italien (Karner et al, 1973; Florineth, 1974) l'Ötztal autrichien (Schiechtl, 1970; Wagner, 1971;
Mayer, 1977) l'Engadine suisse (BraunBlanquet et al, 1954) et la Maurienne française (Bartoli, 1966; Fournier et Peltier, 1987) s'en rapprochent.

Dans les Alpes internes orientales, le climat beaucoup plus sec limite l'extension des sapinières et des pessières qui se localisent presque exclusivement dans le subalpin inférieur (Cadel, 1980); ce confinement dans un créneau altitudinal nettement plus élevé que dans les massifs périphériques tend à atténuer les différences liées au substrat. II en résulte une grande homogénéité des sapinières et des pessières; elles n'occupent qu'une surface réduite par rapport aux autres formations forestières du même niveau (mélézeins et pinèdes).

Si le sapin et l'épicéa occupent des altitudes plus élevées dans les Alpes internes que dans les Alpes externes, leur comportement n'est pas le même dans les 2 soussecteurs.

Dans les Alpes internes occidentales, les forêts du montagnard et du subalpin inférieur d'ubac occupent de vastes surfaces mais présentent des différences physionomiques très marquées. Dans la vallée du Vénéon, la pessière subalpine atteint 1 $850 \mathrm{~m}$; la strate arborescente est monospécifique (Oberlinkels, 1983). Dans la haute Romanche, seul le mélèze est présent; sapins et épicéas sont absents. Dans le haut Queyras, le sapin règne en maître et s'élève jusqu'à $1900 \mathrm{~m}$.

Dans les Alpes internes est (Briançonnais en grande partie, Queyras calcaire) la situation est autre. La grande xéricité climatique et plus encore édaphique induit un comportement différent des 2 essences. Le sapin se localise entre 1600 et $1900 \mathrm{~m}$; il est donc presque entièrement exclu de l'étage montagnard et se réfugie dans le subalpin inférieur. Dans cette tranche altitudinale, grâce à un enracinement profond, il trouve une alimentation en 
eau suffisante. Les effectifs devraient augmenter au cours des prochaines décennies, dans la mesure où l'homme ne favorise plus le mélèze (Cadel, 1980). Quant à l'épicéa, sa place est réduite (Cadel et Gilot, 1963), contrairement à ce qu'on observe dans les Alpes internes septentrionales (Gensac, 1970; Ozenda et Wagner, 1975) qu'elles soient françaises (Gensac, 1967), suisses (Bach et al, 1954; Ellenberg et Klotzli, 1972) ou autrichiennes (Wagner, 1971; Mayer, 1974). Cette faible représentation est plus due à une impossibilité écologique qu'à une absence chorologique. Dans la vallée de la Clarée où sapin et épicéa coexistent, ce dernier n'arrive jamais à concurrencer le sapin; il ne forme une pessière pure qu'au-dessus de la limite thermique de la sapinière (Cadel, 1976). A cause de son enracinement superficiel, l'épicéa est donc moins apte que le sapin à trouver des stations refuges où l'alimentation hydrique en profondeur soit suffisante. Dans les Alpes internes est du Dauphiné (Briançonnais et Queyras), quelques pessières dépérissantes se localisent entre 1850 et $2000 \mathrm{~m}$. Ces observations concernant le comportement de ces 2 essences majeures vont également dans le sens d'une individualisation des 2 sous-secteurs.

\section{La productivité des sapinières et des sapinières-pessières dans les 5 entités phytogéographiques}

On peut se demander si la partition des Alpes dauphinoises en 5 entités phytogéographiques, effectuée à partir de critères floristiques et sociologiques peut être val: dée en prenant des critères de fonctionnement. Nous prendrons comme descripteur la production brute par hectare et par an, telle qu'elle est évaluée par l'Office national des forêts. Elle a été calculée pour les sapinières et les sapinières-pessières en ne prenant en compte que les 2 résineux (Oberlinkels, 1987). Les données ont été fournies par l'ONF. Une critique fondamentale de cette approche comparative réside dans le fait que la valeur de la productivité n'est pas assortie d'une évaluation de la fertilité stationnelle. Néanmoins, on peut tirer quelques enseignements. La productivité diminue de façon régulière d'ouest en est (fig 7). Dans les Alpes externes, elle est toujours supérieure à $4 \mathrm{~m}^{3} \cdot \mathrm{ha}^{-1} \cdot \mathrm{an}^{-1}$ et peut atteindre $7,5 \mathrm{~m}^{3}$. Cette grande amplitude montre le poids des conditions stationnelles.

Dans les Alpes intermédiaires, les valeurs rendent compte des conditions écologiques moins favorables que dans les massifs externes mais nettement moins sévères que dans le secteur intra-alpin. Les différences sensibles entre soussecteur occidental et sous-secteur oriental justifient une individualisation qui, comme nous l'avons vu, est moins évidente sur la base de critères sociologiques. Dans les conditions les plus défavorables du sous-

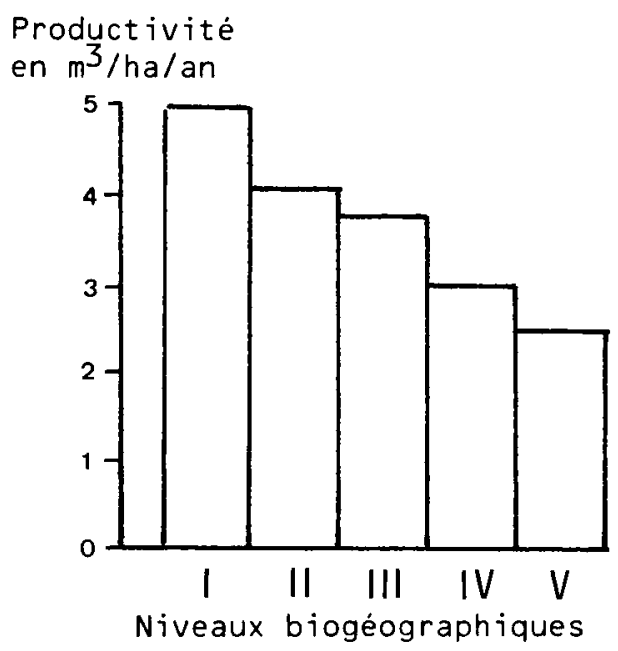

Fig 7. Productivité des sapinières dans les 5 niveaux biogéographiques retenus. 
secteur oriental de ces Alpes intermédiaires la productivité se rapproche des valeurs caractérisant les Alpes internes. On soulignera les chiffres très faibles pour les sapinières du sous-secteur interne est $\left(2,5 \mathrm{~m}^{3} \cdot \mathrm{ha}^{-1} \cdot \mathrm{an}^{-1}\right.$ en moyenne). Ces chiffres modestes ne s'appliquent qu'aux sapinières. Dans l'environnement climatique du Briançonnais, des essences comme le pin à crochet ou le mélèze sont beaucoup plus performantes.

\section{CONCLUSION}

L'analyse statistique que nous avons menée montre qu'il est plus facile d'établir des limites dans les régions marginales où les paramètres écologiques passent par des valeurs extrêmes. Ainsi, en testant l'affinité biogéographique des espèces choisies, l'information mutuelle fait apparaître une partition en deux groupes principaux de taxons : ceux qui sont attirés par la zone externe et repoussent fortement la zone interne et ceux qui ont un comportement inverse. Les Alpes intermédiaires constituent une zone de transition où seul un petit nombre d'espèces trouvent des conditions optimales. II est donc difficile, au seul examen des profils indicés, de justifier le découpage des Alpes dauphinoises en 5 niveaux. Si on prend en compte des critères de type sociologique, des arguments peuvent être trouvés pour valider ce découpage.

La présence de forêts mésophiles de Carpinus betulus, de forêts de bois durs (Pruno-Fraxinetum et Querco-UImetum), de hêtraies très richement pourvues en représentants des Fagetalia (AsperuloFagetum, Cardamino-Fagetum, Fagetum sylvaticae) caractérisent les Alpes externes.

La disparition de ces groupements mais aussi l'implantation de la sapinière- pessière de type Abietetum albae et l'épanouissement de hêtraies-pinèdes, de pinèdes et même de pinèdes-pessières (Pautou et al, 1986) justifient l'individualisation en Oisans d'un secteur des Alpes intermédiaires. La différenciation de 2 sous-secteurs est plus délicate; cependant, dans le sous-secteur occidental, les hêtraies dominent dans le montagnard d'ubac sur calcaire alors que dans le soussecteur oriental ce sont les pinèdes et les pessières.

Les discontinuités de type édaphique que l'on observe depuis la Chartreuse jusqu'au Briançonnais peuvent compliquer l'interprétation phytogéographique que l'on peut faire en se basant sur la distribution des différents taxons. Les facteurs édaphiques peuvent avoir des effets antagonistes sur la distribution des groupements végétaux. Ils peuvent avoir un effet homogénéisateur en permettant l'implantation de types de peuplements voisins dans des situations climatiques différentes : pinèdes mésophiles sur calcaire. Le cas des mégaphorbiaies est intéressant. C'est dans les Alpes intermédiaires qu'elles occupent les plus vastes surfaces. Elles sont rares dans la partie calcaire des Alpes externes malgré la très forte pluviosité. Dans le Briançonnais, c'est la conjonction de facteurs climatiques (précipitations faibles) et de facteurs édaphiques (sols filtrants sur calcaire ou sur quartzite) qui explique leur totale disparition.

La présence de calcaires durs, pauvres en argile, empêchant la formation d'un complexe argilo-humique, favorise inversement la genèse d'un humus peu actif de type moder calcique et on peut observer une forte représentation des plantes de moder aux 2 extrémités du transect. C'est le cas dans les Préalpes sur les calcaires urgoniens et dans le Briançonnais sur les calcaires triasiques.

Depuis les Alpes externes jusqu'aux Alpes internes, sapinières et pessières 
progressent en altitude plus que les étages de végétation (300 $\mathrm{m}$ au lieu de 150). Une des conséquences est que les espèces d'affinité subalpine sont donc plus fréquentes dans les sapinières et les pessières internes que dans leurs homologues externes; nous citerons Rhododendron ferrugineum, Homogyne alpina, Soldanella alpina, Pinus uncinata.

S'il existe des possibilités d'installation de la sapinière tout au long du transect dauphinois, les biotopes favorables deviennent très localisés au fur et à mesure que les précipitations diminuent. Dans le Briançonnais, la sapinière n'est possible que dans des limites d'altitude, d'exposition, d'alimentation hydrique du sol très étroites. En passant des Alpes externes aux Alpes internes, la signification de la sapinière comme climax climatique diminue. Alors que dans la région grenobloise la sapinière peut occuper tous les ubacs entre 800 et $1600 \mathrm{~m}$, dans le Briançonnais elle est localisée dans une faible tranche altitudinale entre 1600 et $1900 \mathrm{~m}$ et sur les versants s'écartant très peu du plein nord.

Dans les Alpes intermédiaires, quelques énigmes restent à élucider. C'est le cas des hêtraies, qui à $10 \mathrm{~km}$ de leur limite orientale constituent, dans le bas Vénéon et dans le Valgaudemar sur des adrets acides, des peuplements purs alors qu'elles sont sporadiques voire absentes dans les ubacs; peut-on expliquer cette absence comme une conséquence des interventions humaines?

La disparition totale du hêtre dans les Alpes internes et l'implantation massive du mélèze représentent 2 caractéristiques majeures de cette zone. La différenciation entre un sous-secteur occidental et un sous-secteur oriental est nettement plus fondée que pour les Alpes intermédiaires. La présence du Piceetum montanum, du Corylo-Populetum, du Salicetum pentan- drae (Braun-Blanquet, 1961), de mélézeins à mégaphorbiaies et de pessières subalpines montre les affinités entre ce que nous appelons les Alpes internes occidentales et d'autre part les Alpes internes autrichiennes et suisses. Quelques problèmes demeurent dans ce sous-secteur ouest : pourquoi l'épicéa si abondant dans la vallée du Vénéon est-il absent de la vallée de la Romanche à l'amont du barrage du Chambon et inversement pour le mélèze ? Est-ce le reflet de voies de migrations non achevées ou $y$-a-t-il d'autres explications?

\section{RÉFÉRENCES}

Bach R, Kuoch R, lberg R (1954) Wälder der Schweizer Alpen im Verbreitungsgebiet der Weisztanne. Entscheidende Standorstsfaktoren und Böden. Mitt Schweiz Anst Forst Vers $30,261-314$

Bartoli C (1962) Premières notes sur les associations forestières du massif de la Grande Chartreuse. Ann Ecole Nat Eaux For 19, 357-377

Bartoli C (1966) Études écologiques sur les associations forestières de la Haute-Maurienne. Ann Sci For 23, 433-751

Braun-Blanquet J (1961) Die inneralpine Trockenvegetation. G Fischer, Stuttgart

Braun-Blanquet J, Pallmann H, Bach R (1954) Vegetation und Böden der Wald- und Zwergstranchgesellschaften (Vaccinio-Piceetalia). In: Résultats des recherches scientifiques entreprises au Parc National Suisse IV, $1-198$

Cadel G (1976) La Névachie : son intérêt botanique. Rapport pour le Parc National des Ecrins, $16 p, 1$ carte

Cadel G (1980) Séries de végétation et sols du Subalpin briançonnais sur roches-mères silicoalumineuses. Comparaison avec la Maurienne et la Tarentaise. Sci Sol 4, 249-264

Cadel G, Gilot JC (1963) Feuille de Briançon (XXXV-36). Doc Carte Vég Alpes 1, 9ł-139 
Cadel G, Pautou G (1984) Les groupements forestiers des Alpes intermédiaires dauphinoises : particularités biogéographiques, phytosociologiques et écologiques. Colloque Ecologie des milieux montagnards et de haute altitude. Doc d'Ecol pyrénéenne III-IV, 21-27

Coûteaux M (1981) Caractérisation pollenanalytique en Oisans des milieux forestiers et des milieux supraforestiers dans l'état actuel et dans le passé. CR colloque, La limite supérieure de la forêt et sa valeur de seuil. Perpignan, 139-158

Daget P, Godron M, Guillerm JL (1972) Profils écologiques et information mutuelle entre espèces et facteurs écologiques. Actes du Symp Int Grunfugen und Methoden in der Pflanzensoziologie Rinteln, 1970, 121-149

Duchaufour P, Fourchy $P$ (1952) Étude sur l'écologie et la sylviculture du mélèze. Pédologie et facteur biotiques. Ann ENEF, XIII, 139-195

Ellenberg G, Klotzli $F$ (1972) Waldgesellschatten und Waldsthandorte der Schweiz. Mém Inst Suisse Rech For 48, 589-930

Faure C (1968) Feuille de Vif (XXXII-35). DoC Carte Vég Alpes, VI, 7-69

Fénélon JP (1982) Qu'est-ce que l'analyse des données. Lefonen, Paris, $311 \mathrm{p}$

Ferrand $T$ (1984) Étude d'une séquence bioclimatique (sol, végétation) sur amphibolites : Massif de Belledonne, Taillefer. Mémoire de DEA Biol Physiol Pédo Nancy et USMG, $88 p$

Florineth $F$ (1974) Vegetation und Böden des oberen Vinschgaues (Südtirol, Italien); Ber Naturwiss Med Ver Innsb 61, 43-70

Fournier J, Peltier JP (1987) Les secteurs biogéographiques de la Maurienne (Savoie) : leur délimitation par f'étude des précipitations et des groupements végétaux forestiers. Doc Cart Ecol XXX, 3-24

Gauthier B, Godron M, Herniaux P, Lepart J (1977) Un type complémentaire de profil écologique : le profil écologique indicé. Can $J$ Bot 2859-2865

Gensac P (1967) Les forêts d'Epicéa de Moyenne Tarentaise. Recherche des diffé- rents types de pessières. Rev Gén Bot 74 425-528

Gensac P (1970) Les pessières de Tarentaise comparées aux autres Pessières alpestres. Ber Geobot Inst Eidg Tech Hochsch Stif Ruebel Zuer 43, 104-139

Gillot $P$ (1981) Le montagnard et le subalpin d'adret dans les Alpes intermédiaires dauphinoises. Mérnoire de DEA d'Écologie appliquée, Grenoble, $133 p$

Karner A, Kral F, Mayer H (1973) Das inneralpine Vorkommen der Tanne im Vinschgau. Zentralblat Gesamte Forstwes 90, 129-163

Kuoch R (1954) Wälder der Schweizer Alpen im Verbreitungsgebiet der Weisstanne. Ann Inst Fed Rech For 30, 131-260

Lacoste A (1972) La végétation de l'étage subalpin du bassin supérieur de la Tinée (Alpes Maritimes). Applications de l'analyse multidimensionnelle aux données floristiques et écologiques. Thèse Paris-Orsay, $295 \mathrm{p}$

Mayer H (1963) Tannenseich Wälder am Nordabfall der mitteeren Ostalpen. BLV, Munich, $208 \mathrm{p}$

Mayer $H$ (1974) Wälder des Ostalpenraumes. Fischer, Stuttgart, $344 \mathrm{p}$

Mayer $H$ (1977) Waldbauliche Untersuchungen in Lärchen-Zirbenwäldern der Ötztaler Alpen. Zentralbl Gesamte Forstwes Wien 94, 11-32

Meyer D (1981) La végétation des vallées de Vallouise, du Fournel et de la Biaysse (Pelvoux oriental, Haute-Alpes). Analyse phytosociologique et phytogéographique des átages collinéen, montagnard et subalpin. Thèse $3^{\circ}$ cycle Univ Aix-Marseille I, $176 p$

Oberlinkels M (1983) Les groupements forestiers d'ubac de Bourg d'Oisans à StChristophe en Oisans; contribution à l'étude d'un transect dans les Alpes dauphinoises. Mém DEA Ecol Appl USMG, 36 p

Oberlinkels M (1987) Étude phytoécologique des vallées occidentales du massif des Ecrins. Thèse Univ Grenoble, $123 \mathrm{p}$

Ozenda $P$ (1966) Perspectives nouvelles dans l'étude phytogéographique des Alpes du Sud. Doc Carte Vég Alpes, VI:1-198, 3 cartes coul 
Ozenda P (1985) La végétation de la chaine alpine dans l'espace montagnard européen. Masson, Paris, $330 p$

Ozenda P, Wagner H (1975) Les séries de végétation de la chaine alpine et leurs équivalences dans les autres systèmes géographiques. Doc Cartogr Ecol XVI, 49-64

Ozenda $P$, Cadel G (1981) Analyse et cartographie des groupements végétaux de la Guisane (Hautes-Alpes). Recherches en Briançonnais. Actions concertées DGRST, CEMAGREF, 5-18
Pautou G, Cadel G, Girel J (1986) Carte des peuplements forestiers du bassin de Bourg d'Oisans à 1:25 000. Doc Cart Ecol

Schiechtl HM (1970) Die Ermittlung der Potentiellen Zirbenwaldfläche im Ötztal. Mitt Ostalp Din Ges Vegetationskunde 11, 197-204

Tonnel A, Ozenda $P$ (1964) Séries de végétation de la moitié sud du département de I'Isère. Doc Carte Vég Alpes II, 9-35

Wagner $\mathrm{H}$ (197;) Natürliche Vegetation. In: Osterreich-Atlas IV/3 